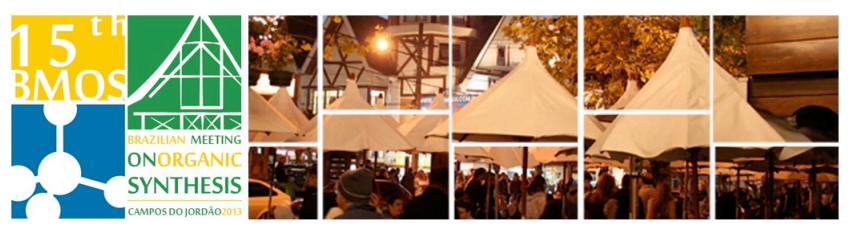

\title{
Catalytic oxidations by metal nanoparticles: Pd, Au and AuPd core-shell nanoparticle catalysts
}

\author{
Liane M. Rossi, ${ }^{1 *}$ Tiago A. G. Silva, ${ }^{1}$ Erico Teixeira-Neto, ${ }^{1}$ Núria Lopez ${ }^{2}$ \\ ${ }^{1}$ Universidade de São Paulo, São Paulo 05508-000, Brazil
}

2 Institute of Chemical Research of Catalonia, ICIQ, Tarragona 43007, Spain

*e-mail corresponding author: Irossi@iq.usp.br

Keywords: gold, palladium, bimetallic nanoparticles

\section{Abstract Speech}

Selective aerobic oxidations are among the most important transformations in the manufacture of fine chemicals. Traditionally, these processes use stoichiometric reagents, such as permanganate and dichromate, and end up with a large amount of toxic waste. Currently, there is considerable pressure to replace these technologies with cleaner catalytic alternatives. $\mathrm{Pd}, \mathrm{Au}$ and AuPd bimetallic nanoparticles have received special attention for their catalytic properties in oxidations. Bimetallic nanoparticles may have metallic domain distributions ranging from homogeneous alloys to core-shell nanoparticles. The production of different nanostructured morphologies depends on the synthetic protocol, miscibility of both metals and post-synthesis treatment. Palladium and gold are miscible in almost all compositions, forming AuPd alloy nanoparticles. However, it has been shown that $\mathrm{Pd}$ segregates to the surface upon calcination producing alloy nanoparticles with a Pd-rich shell surrounding an Au-rich core [1]. Taking into account this behavior, we have designed core-shell nanoparticles prepared by successive reduction of palladium over preformed gold nanoparticles. Monometallic Au and Pd catalysts and bimetallic AuPd catalysts containing various amounts of palladium were compared in the oxidation of benzyl alcohol, a model substrate for oxidation reactions. A marked influence of the amount of palladium (shell metal) on the catalytic activity of an existing Au catalyst was observed. The monometallic $\mathrm{Pd}$ catalyst is also active, but less selective than the bimetallic AuPd systems. The monometallic Au catalyst is not active, unless a base is present.

In the catalytic studies, the bimetallic AuPd catalysts have a volcano-like behavior characterized by a steady increase in benzyl alcohol conversion as the quantity of added $\mathrm{Pd}$ (shell metal) was increased relative to Au (core metal), with a consistent decrease in conversion when the $\mathrm{Pd}$ composition was increased to greater than $10 \mathrm{~mol} \%$. If the $\mathrm{Pd}$ atoms are primarily on the surface of $\mathrm{Au}$, as observed in the morphological studies, the composition of the most active catalyst (89.9\% $\mathrm{Au}$ and $9.1 \% \mathrm{Pd}$ ) correlates with the composition required for the complete coverage of Au cores $(\mathrm{d}=12.0 \pm 3.2 \mathrm{~nm})$ with one atomic layer of $\mathrm{Pd}$. This catalyst was investigated by $\mathrm{X}$ ray energy dispersive spectroscopy in a scanning transmission electron microscope (XEDS-STEM) to determine both the compositional distribution between particles and the existence of compositional rich domains within each particle. Density Functional Theory identify that the volcano-like behavior comes from the delicate balance between the number of active sites and the easy product desorption. Both properties are extremely sensitive to the Pd content.

We elaborate experimental and theoretical insights on improving the activity and selectivity of AuPd NPs by tailoring the best electronic and geometric properties for the selective oxidation of alcohols. This finding shows that many opportunities remain to obtain enhanced properties in bimetallic nanomaterials by tailoring the amount of each metal and the metal domain distribution, and opens new possibilities in the field of nanomaterials synthesis and design.

This work was supported by the Brazilian agencies CAPES, CNPq, INCT-Catalise and FAPESP.

\section{REFERENCES}

D.I. Enache, et al., Science, 311, 362 (2006).3335. 